\title{
Hierarchical Control and Skilled Typing: Evidence for Word-Level Control Over the Execution of Individual Keystrokes
}

\author{
Matthew J. C. Crump and Gordon D. Logan \\ Vanderbilt University
}

\begin{abstract}
Routine actions are commonly assumed to be controlled by hierarchically organized processes and representations. In the domain of typing theories, word-level information is assumed to activate the constituent keystrokes required to type each letter in a word. We tested this assumption directly using a novel single-letter probe technique. Subjects were primed with a visual or auditory word or a visually presented random consonant string and then probed to type a single letter from the prime or another randomly selected letter. Relative to randomly selected letters, probe responses were speeded for first, middle, and last letters contained in visual and auditory word primes but not for middle and last letters contained in random consonant primes. This suggests that word-level information causes parallel activation of constituent keystrokes, consistent with hierarchical processing. The role of hierarchical processing in typing and routine action is discussed.
\end{abstract}

Keywords: typing, hierarchical processing, performance, routine, motor control

Everyday tasks and routines are structured hierarchically with higher level goal completion resting on the completion of component subgoals. For example, the higher level goal of hammering a nail involves several lower level strokes of the hammer on the head of the nail until the nail head is flush with the board. Theories of routine action commonly assume that the hierarchical structure of tasks is reflected in the cognitive processes and representations mediating task performance (Miller, Galanter, \& Pribram, 1986), whereby higher level goal representations control the unpacking and execution of constituent lower level goal representations. In the present experiments, we sought to test how higher level goals interact with lower level goals in the domain of skilled typing. In particular, typing models assume that word-level representations activate keystroke execution processes for each letter in the word in parallel. In three experiments, we developed a novel singleletter probe technique to test this assumption of parallel activation in skilled typists.

Typing is a compelling example of the ability to fluently and rapidly coordinate intention with performance (Shaffer, 1976). The intention to type and perform keystrokes is argued to be organized hierarchically with higher level intentions to type words and sentences controlling lower level processes through which words are converted into ordered keystrokes (Shaffer, 1975). We have elaborated on these assumptions in our inner-outer loop theory of typing (Logan \& Crump, 2009). We characterized the outer loop as receiving input from the world in the form of written or spoken

This article was published Online First October 4, 2010

Matthew J. C. Crump and Gordon D. Logan, Department of Psychology, Vanderbilt University.

This research was supported by National Science Foundation Grant BCS 0646588.

Correspondence concerning this article should be addressed to Matthew J. C. Crump, Department of Psychology, Vanderbilt University, Nashville TN 37203. E-mail: matt.crump@vanderbilt.edu language and generating a series of words that are passed individually to the inner loop. The inner loop receives words as input, translates each word into keystrokes corresponding to each letter, and then executes them. Our previous work focused on the role of attention and monitoring during keystroke execution to investigate the assumption that outer and inner loop processes operate in an encapsulated fashion. We assumed that the outer loop does not know the details of keystroke execution that are controlled by the inner loop, and can only access them indirectly by observing the output of the inner loop. We offered evidence of encapsulation by demonstrating that typing performance is substantially disrupted when typists are instructed to monitor their hand movements while typing. These findings provided insight into the kind of information represented in each loop but not on how the outer loop controls inner loop processing. In the current set of experiments, we focused on a separate set of issues; that is, how word-level information in the outer loop unpacks letter information inside the inner loop to coordinate keystroke execution.

In further investigating the inner-outer loop theory, we aimed to test hierarchical processing assumptions common to models of typing, which incorporate hierarchical processing assumptions in two steps (John, 1996; Rumelhart \& Norman, 1982; Salthouse, 1986; Wu \& Liu, 2008). First, word-level units are assumed to cause parallel activation of constituent keystrokes for each letter in the word. Second, a serial control process is assumed to specify keystroke ordering. The focus of our current experiments was to test the assumption that words cause parallel activation of the processes controlling keystroke execution for each letter in the word. This assumption is consistent with a number of findings in the typing literature, but it has not been directly tested. We employed a novel single-letter probe technique to determine the extent to which word information facilitates the typing of component letters. Before describing our experiments, we review existing findings highlighting the importance of words as a unit of control 
in typing and findings consistent with the assumption that words activate keystrokes in parallel.

There are at least two lines of evidence suggesting that words are an important unit of control in typing. First, copy-typing rate depends on the wordlike structure of the to-be-typed text. In particular, words are typed much faster than strings of random letters (Larochelle, 1983; Shaffer \& Hardwick, 1968; Sternberg, Knoll, \& Wright, 1978). Typing speed does not depend on meaningful sequences of words, as typing speed does not deteriorate for texts containing randomly arranged words (Shaffer \& Hardwick, 1968). These findings indicate that structures within words (e.g., bigrams, trigrams) and words themselves play an important role in the control of typing ability.

Second, typing is dependent on reading and language abilities, which employ words as the most meaningful unit. Speaking anecdotally, typists commonly report silently thinking the words they are typing. From an empirical standpoint, several studies have demonstrated that typing speed is influenced by boundaries corresponding to linguistic units. For example, typing speed is influenced by syllable boundaries within words, with slower interkeystroke intervals (IKSIs) between syllable boundaries than within syllable boundaries (Weingarten, Nottbusch, \& Will, 2004; Will, Nottbusch, \& Weingarten, 2006). These findings suggest that word-level representations developed in the context of reading and language domains are exploited during typing.

There are at least two lines of evidence suggesting that words activate to-be-typed letters in parallel. First, the notion of parallel activation is consistent with kinematic findings (Gentner, 1983). For example, typing rate is much faster when letter sequences require hand alternations as opposed to hand repetitions. Typing rate is further slowed when letter sequences require within finger repetitions. These findings are consistent with the notion that letters beyond the current letter being typed are activated. Furthermore, high-speed video recordings of finger movements during typing reveal that skilled typists' fingers move in parallel to specific letters during typing of a word (Gentner, Grudin, \& Conway, 1980). Last, keystroke kinematics (e.g., translational and rotational motion) for typing letters on one hand can operate independently of contralateral hand movement patterns (Flanders \& Soechting, 1992), indicating that parallel control of hand motion during typing is possible.

Second, one can elicit Simon-like interference effects (for a review, see Lu \& Proctor, 1995) in typing by presenting words on the left or right side of the screen. Simon effects refer to the finding that spatial compatibility between target and response locations interferes with performance. For example, Logan (2003) found that words typed entirely with the left (or right) hand were typed more rapidly when they were presented on the left (or right) side of the screen than when they were typed with the hand on the opposite side, suggesting that spatial codes from the stimulus activated spatial codes for the responses. This effect disappeared when the first letter of the word was typed with one hand and the remaining letters were typed with the other hand, suggesting that the spatial codes for the subsequent letters balanced the spatial code for the first letter and eliminated the Simon effect. This balancing of spatial codes suggests that letters beyond the first were activated in parallel with the first letter.

In sum, previous research has demonstrated that words are important units in typing and that words may activate letters in parallel; however, these findings have not provided a direct test of the parallel activation assumption. Although it is clear that wordlevel representations are important in typing skill, there is little direct evidence of functional connections between word-level and letter-level representations.

Our experiments were designed to determine whether words activate keystrokes for their constituent letters in parallel. To test this assumption, we created a novel single-letter probe technique. Subjects were briefly primed with a visually presented word (Experiment 1 ), auditory word (Experiment 2 ), or visually presented random letter string (Experiment 3) that was followed by a probe, which the subjects had to type as quickly and accurately as possible. The probe was either a complete repetition of the prime (e.g., a full word) or a single letter. For single-letter probes, the letter was either primed (the first, middle, or last letter of the prime word) or unprimed (another randomly chosen letter that was not presented in the prime). If words activate their constituent letters in parallel, response time (RT) for primed probes should be faster than RT for unprimed probes, a pattern we term the word advantage effect.

Across experiments, we asked whether word advantage effects reflect the influence of word-level representations on keystroke execution or merely the perception of particular letters. The presentation of a visual word prime entails the presentation of the letters composing the word. As a result, the word advantage effect could be driven solely by the presentation of individual letters. We controlled for the influence of letter-level influences across experiments in two ways. First, in Experiment 2, we employed auditory primes, which present word information but do not present visual information about particular letters. If word advantage effects are entirely driven by the visual presentation of letters, then we would not expect auditory primes to produce such a pattern. Second, in Experiment 3, we employed random consonant strings, which present letter information in the absence of word information. If word advantage effects are entirely driven by the visual presentation of letters, then we would expect an advantage in RT for random consonant strings, even though they do not carry word information. Alternatively, if word-level representations do activate constituent letters, then we would expect word advantage effects for both visual and auditory word primes but no word advantage effect for random consonant string primes.

Responses to word probes allowed converging tests of the importance of word-level representations in skilled typing ability in measures of first keypress RT and IKSI. If word-level representations support skilled typing (Larochelle, 1983; Shaffer \& Hardwick, 1968; Sternberg, Knoll, \& Wright, 1978), then RTs and IKSIs should be faster when words are typed (Experiments 1 and 2) than when nonwords are typed (Experiment 3).

\section{Experiment 1: Visual Word Primes}

We conducted three versions of Experiment 1 to determine whether priming was affected by strategic factors (Neely, 1977; for a review, see McNamara, 2005). We assessed strategic factors by manipulating the proportion of full-word (five- and seven-letter words) and single-letter probes in a between-subjects design with three groups. For one group, the proportion of full-word probes was high $(p=.72)$, which allowed subjects to develop the expectation that the probe would usually be a full word. For the second 
group, the proportion of full-word probes was low $(p=.28)$, which allowed subjects to develop the expectation that the probe would usually be a single letter. In both experiments, when the probe was a single letter, there was an equal likelihood that the subjects would receive a first-, middle-, last-, or other-letter probe. As a result, single-letter probes were more likely to be primed than unprimed, and subjects may have employed a strategy to optimize responding to within-word letter probes, which could explain the word advantage effects. For the last group of subjects, we equated the proportion of primed and unprimed single-letter probes. The design was a low-proportion full- probe condition $(p=.28)$; however, the proportion of primed single-letter probes was $.36(.12$ for each first-, middle-, and last-letter condition), and the proportion of unprimed single-letter probes was .36. The latter group was termed the low-proportion full-word equal group.

If word representations activate keystrokes for constituent letters in parallel, then we expect RTs to be shorter for primed single-letter probes (e.g., first, middle, last) than unprimed singleletter probes (e.g., other letters). Failure to find a word advantage effect for primed single-letter probes would suggest that word representations do not activate constituent keystrokes for letters in the word in parallel.

\section{Method}

Subjects. Subjects were 48 students (16 in each proportion condition) from Vanderbilt University, recruited for the selfreported ability to type 40 words per minute (wpm) or better. Their skill was confirmed on a typing test. Typing test performance was as follows: high-proportion full-word group was $51 \mathrm{wpm}$ (range: 33-78), mean full-word error rate was .08 (range: .01-.16); lowproportion full-word group was $71 \mathrm{wpm}$ (range: 55-95), mean error rate was .07 (range: .02-.12); and low-proportion full-word equal group was $71 \mathrm{wpm}$ (range: $36-103$ ), mean error rate was .08 (range: .03-.13). All subjects were compensated with course credit or were paid $\$ 12$ per $1 \mathrm{hr}$ of participation. All subjects had normal or corrected-to-normal vision and spoke English as a first language.

Apparatus and stimuli. The experiment was conducted on a personal computer with a 15 -in. $(38.1-\mathrm{cm})$ super video graphics array (SVGA) monitor running in-house software controlled by MetaCard (MetaCard Corp., Boulder, CO). Typing responses were issued on a standard QWERTY keyboard.

We gathered a set of five- and seven-letter words from the University of South Florida word norms (Nelson, McEvoy, \& Schreiber, 1998). We applied a filter to ensure that the first, middle, and last letter in each word was unique. There were 894 five-letter words and 668 seven-letter words. The mean word frequency (Kucera \& Francis, 1967) for five-letter words was 56 per million, with a range of $0-1,599$. Mean word frequency for seven-letter words was 55 per million, with a range of $0-2,096$. Five-letter words were $66 \%$ one-syllable words, $33 \%$ two-syllable words, and $1 \%$ three-syllable words. Seven-letter words were $1 \%$ one-syllable, $73 \%$ two-syllable, $25 \%$ three-syllable, and $1 \%$ foursyllable words. The relative letter frequency was $4.2 \%$ for primed letters and $4.3 \%$ for unprimed other letters. Primed letters were $34 \%$ home-row keys and $66 \%$ non-home-row keys. Unprimed letters were $36 \%$ home-row keys and $64 \%$ non-home-row keys.
Primed letters were $26 \%$ vowels and $74 \%$ consonants, and unprimed letters were $14 \%$ vowels and $86 \%$ consonants.

Procedure. We employed a variation on the discontinuous typing paradigm (Ostry, 1980; Sternberg, Monsell, Knoll, \& Wright, 1978). Instead of giving subjects a single word to type on each trial, we included a prime stimulus prior to the presentation of the probe stimulus. The design involved two within-subject factors (probe type and word length) and one between subject factor (probe-type proportion). There were five probe types, involving full words or single letters. Full words were always exact repetitions of the prime. Single-letter probes involved the first, middle, or last letter of the prime word, or another randomly selected letter was chosen from the remaining letters in the alphabet.

The proportion of full-word versus single-letter probes was manipulated between subjects across three conditions. In the highproportion full-word probe condition, subjects received $72 \%$ fullword probes and $28 \%$ single-letter probes (7\% for each of the first-, middle-, last-, and other-probe types). There were two lowproportion full-word probe conditions. In the low-proportion fullword group, subjects received $28 \%$ full-word probes and $72 \%$ single-letter probes (18\% across each single-letter probe type). In the low-proportion full-word equal group, subjects received $28 \%$ full-word probes and $72 \%$ single-letter probes (12\% for each first-, middle-, and last-letter probe, and 36\% for unprimed letters). Each experiment involved 800 trials, with 400 unique five-letter word trials and 400 unique seven-letter word trials.

Subjects were seated approximately $57 \mathrm{~cm}$ from the computer monitor. At the beginning of each trial, a fixation cross was presented for $500 \mathrm{~ms}$. The prime word was presented for $150 \mathrm{~ms}$, followed by a 150-ms interstimulus interval during which the screen remained blank. The fixation cross was presented in the center of the screen. To distinguish the prime from the probe, we presented the prime in the same location as the fixation cross in the center of the screen. The probe was presented one line (15 $\mathrm{mm}$ ) below the prime. All letters in the prime and probe were 15 $\mathrm{mm}$ in height, displayed in Helvetica font, and presented in uppercase format. Words were displayed in black on a white background. Subjects were instructed to quickly and accurately type out the probe word or letter and then press the space bar to move on to the next trial. The target word was removed from the screen after subjects pressed the space bar. The next trial was issued automatically $500 \mathrm{~ms}$ later. Subjects were given online feedback. Their typed responses were presented at the bottom of the screen in lowercase. Subjects were instructed to ignore the case of the probe and typed letters. Subjects were further instructed that the backspace key would not function, allowing us to measure keypress errors. Subjects were given a break screen every 100 trials.

At the end of the experiment, each subject was given a typing test to measure his or her average typing speed. The test involved copy-typing one of four short paragraphs (115 words in length) taken from Logan \& Zbrodoff (1998). Wpm was defined as the number of five-letter strings typed per minute.

\section{Results}

Mean RTs and error rates for each probe condition and word length level for each full-word probe proportion condition are displayed graphically in Figure 1. Analyses of variance (ANOVA) tables for all statistical analyses are presented in Table 1. For each 
Five letter RTs and Error rates

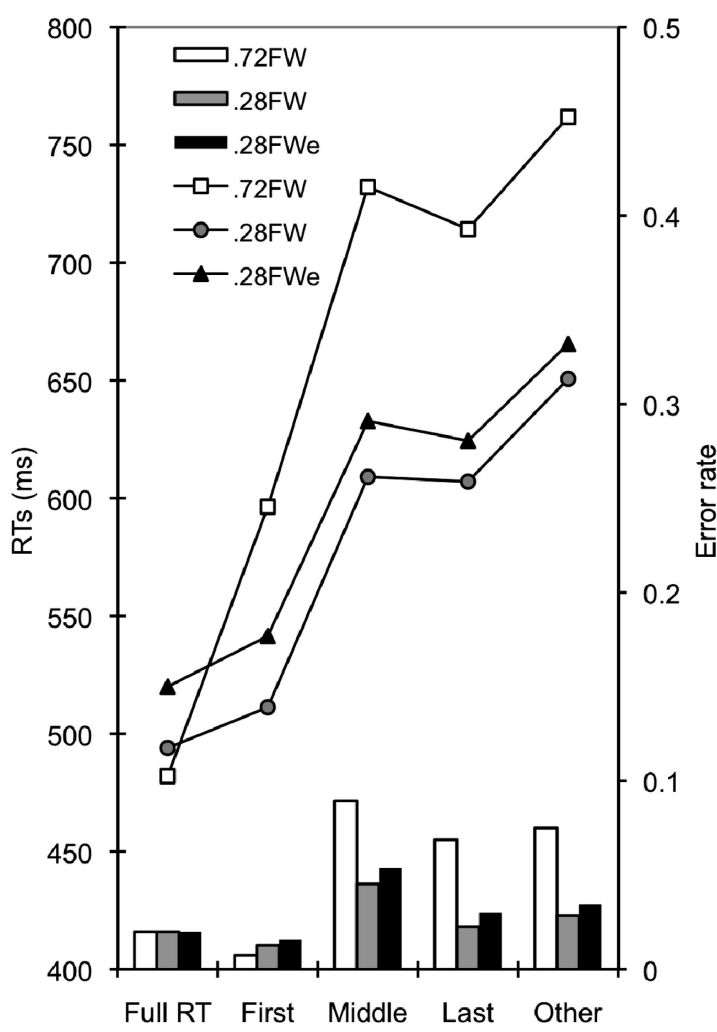

Seven letter RTs and Error rates

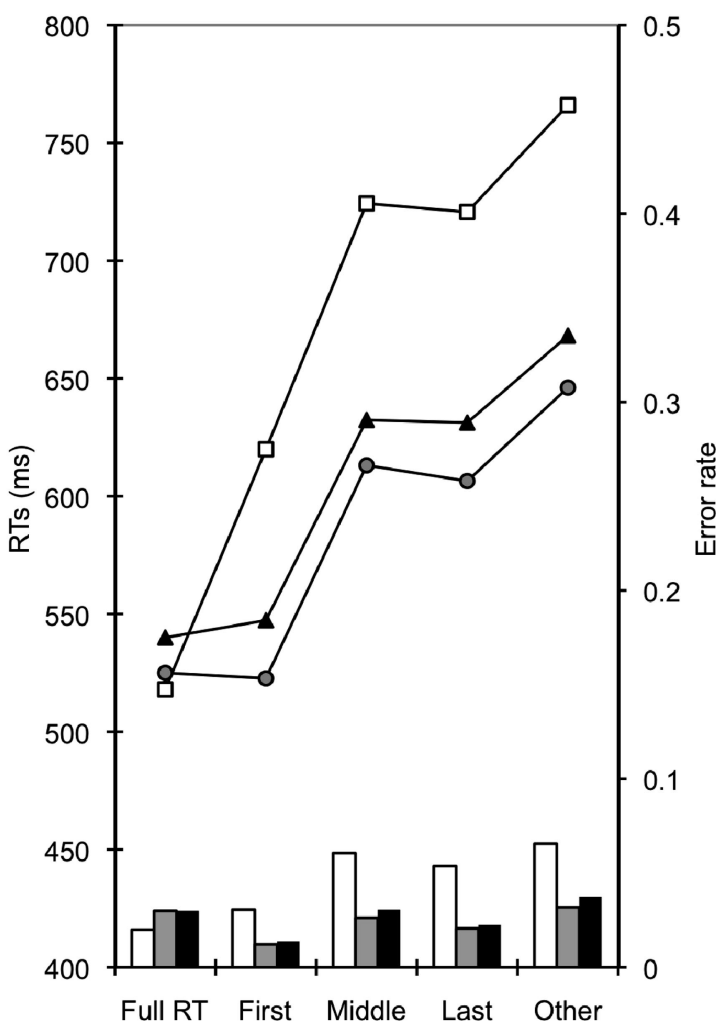

Figure 1. Mean reaction times (RTs) and error rate for full-word and single-letter probes for five- and seven-letter words for each proportion condition in Experiment 1: .72FW refers to the high-proportion full-word probe condition, $.28 \mathrm{FW}$ refers to the low-proportion full-word probe condition, and $.28 \mathrm{FWe}$ refers to the low-proportion full-word probe condition in which equal proportions of both primed and unprimed letters were used. In Experiment 1, visually presented word primes were used.

subject, RTs in each condition were submitted to an outlier analysis (Van Selst \& Jolicoeur, 1994), which trimmed an average of $3 \%$ of the observations. Unless otherwise noted, an alpha criterion of .05 is adopted for all statistical tests.

The primary question was whether primed single-letter probes were typed faster than unprimed single-letter probes. When collapsed across proportion full-word probe conditions, RTs for first letters $(557 \mathrm{~ms})$ were significantly shorter than those for middle $(657 \mathrm{~ms})$ and last $(651 \mathrm{~ms})$ letters. RTs for middle and last letters were both significantly shorter than RTs for other letters $(693 \mathrm{~ms})$. There was no significant difference between RTs for middle and last letters. The pattern of the differences was the same across the probe probability conditions and word lengths; there were no significant interactions. In addition, the three-way Proportion $\times$ Word Length $\times$ Probe Type interaction was not significant. The faster RTs to within-word probes (first, middle, and last letters) than to other probes (not from the word) show the predicted priming effects, which suggest that all the letters in the word primes were activated in parallel.

Although the proportion manipulation did not influence the pattern of single-letter priming effects, it did influence overall RT for the single-letter probes. To examine this finding, we focused on unprimed letter probes. RTs to type other-letter probes were longer in the high-proportion full-word condition $(764 \mathrm{~ms})$ than in the low-proportion full-word condition $(648 \mathrm{~ms}), F(1,135)=145.30$, $M S E=1471.35$; and low-proportion full-word equal condition $(667 \mathrm{~ms}), F(1,135)=102.65, M S E=1471.35$. There was no significant difference between RTs to unprimed letter probes in the low-proportion full-word conditions. Faster RTs in the lowproportion full-word conditions suggest that strategic factors, specifically the general expectation to receive a single-letter probe, influenced the overall speed of responding to single-letter probes.

We performed one additional ANOVA on the RTs for single letters to address potential confounds in the distribution of primed and unprimed letters. In particular, some primed letters $(i, j, q, u$, $\mathrm{v}, \mathrm{w}, \& \mathrm{z}$ ) did not occur in each of the first, middle, and last positions for each subject. As a result, mean RTs for first, middle, and last letters were less likely to contain responses for these letters than the mean RTs for unprimed letters, which included all letters with equal frequency. Given that this letter set contains several low-frequency letters and performance in typing these letters may be slower than normal, it is possible that the RT for unprimed letters was slowed because of a higher proportion of RTs from this letter set. To investigate this potential confound, we repeated the previous analysis and excluded all RTs for each condition that involved responses to the previously mentioned 
Table 1

Experiment 1 Analysis of Variance Tables for Single-Letter Probe Reaction Times and Error Rates

\begin{tabular}{|c|c|c|c|c|c|}
\hline Source & $F$ & $d f \mathrm{~s}$ & $M S E$ & $p$ & $\eta_{\mathrm{p}}^{2}$ \\
\hline \multicolumn{6}{|c|}{ Reaction times } \\
\hline Proportion $(\mathrm{P})$ & 4.47 & 2,45 & 94540.49 & $.02^{*}$ & .17 \\
\hline Length (L) & 3.25 & 1,45 & 545.92 & .08 & .07 \\
\hline$P \times L$ & 0.25 & 2,45 & 545.92 & .78 & .01 \\
\hline Probe & 221.84 & 3,135 & 1471.35 & $.0001^{*}$ & .83 \\
\hline \multicolumn{6}{|l|}{ Linear contrasts } \\
\hline First vs. middle & 331.42 & 1,135 & 1471.35 & $.0001^{*}$ & .71 \\
\hline First vs. last & 289.28 & 1,135 & 1471.35 & $.0001^{*}$ & .68 \\
\hline Middle vs. other & 41.77 & 1,135 & 1471.35 & $.0001^{*}$ & .24 \\
\hline Last vs. other & 58.67 & 1,135 & 1471.35 & $.0001^{*}$ & .30 \\
\hline Middle vs. last & 1.43 & 1,135 & 1471.35 & .28 & .01 \\
\hline $\mathrm{P} \times$ Probe & 1.45 & 6,135 & 1471.35 & .20 & .06 \\
\hline $\mathrm{L} \times$ Probe & 2.31 & 3,135 & 456.80 & .08 & .05 \\
\hline $\mathrm{P} \times \mathrm{L} \times$ Probe & 0.83 & 6,135 & 456.80 & .55 & .04 \\
\hline \multicolumn{6}{|c|}{ Errors } \\
\hline Proportion $(\mathrm{P})$ & 6.22 & 2,45 & 0.007 & $.0001^{*}$ & .22 \\
\hline Length $(\mathrm{L})$ & 4.28 & 1,45 & 0.001 & $.04^{*}$ & .09 \\
\hline$P \times L$ & 0.51 & 2,45 & 0.001 & .61 & .02 \\
\hline Probe & 16.73 & 3,135 & 0.001 & $.00^{*}$ & .27 \\
\hline $\mathrm{P} \times$ Probe & 2.35 & 6,135 & 0.001 & $.03^{*}$ & .09 \\
\hline $\mathrm{L} \times$ Probe & 4.09 & 3,135 & 0.001 & $.01^{*}$ & .08 \\
\hline $\mathrm{P} \times \mathrm{L} \times$ Probe & 0.98 & 6,135 & 0.001 & .44 & .04 \\
\hline
\end{tabular}

${ }^{*} p<.05$.

letter set. More precisely, we only included letters that did occur in each of the first, middle, and last positions for each subject. The pattern and significance of the first-, middle-, and last-letter advantage effects remained the same. First letter RTs $(553 \mathrm{~ms})$ were faster than RTs for middle $(647 \mathrm{~ms}), F(1,135)=318.28, M S E=$ $659.65, \eta_{\mathrm{p}}^{2}=.70$, and last letters $(650 \mathrm{~ms}), F(1,135)=342.89$, $M S E=659.65$. RTs for middle and last letters were not significantly different from one another $(F<1)$. RTs for middle, $F(1$, $35)=19.57, M S E=659.65$, and last letters, $F(1,135)=14.03$, $M S E=659.65$, were both significantly faster than RTs for other letters $(670 \mathrm{~ms})$.

Error rates for single-letter probes were submitted to a 3 (fullword probe proportion: high, low, and low equal full) $\times 2$ (word length: five vs. seven) mixed design ANOVA, reported in Table 1. There were no significant differences in error rates across proportion conditions or as a function of probe word length. These findings indicate that the RT differences for single-letter probes were not driven by speed-accuracy trade-offs.

We analyzed full-word typing performance in terms of RT, IKSI, and error rate. The first-key-press RT refers to the amount of time elapsed between the onset of the probe and keystroke to the first letter, the IKSI, referring to the slope of a linear function relating the time at which each keystroke occurred to the position of the keystroke in the word, and the error rate was defined conservatively to include any cases in which the entire word was typed incorrectly.

We compared performance on full-word probes across proportion conditions. Statistical analyses for full-word probes for all experiments are reported in Table 2. Planned comparisons revealed no significant differences between first-key-press RTs in the highproportion full-word probe $(500 \mathrm{~ms})$, low-proportion full-word probe $(509 \mathrm{~ms})$, and low-proportion full-word equal $(530 \mathrm{~ms})$ groups. Similarly, there were no significant differences in IKSIs in the high $(157 \mathrm{~ms})$, low $(151 \mathrm{~ms})$, and low equal $(151 \mathrm{~ms})$ full-word probe-proportion groups (see Figure 2). These findings suggest that full-word typing performance was strategy invariant and imply that word typing processes were highly automatized (LaBerge \& Samuels, 1974).

\section{Discussion}

In Experiment 1, we found RT advantages for first-, middle-, and last-letter probes relative to other-letter probes, which suggest that word primes activate keystrokes for all letters in the word in parallel. The pattern did not vary as a function of word length or probe probability.

Full-word probe probability did slow single-letter typing. Overall, single-letter RTs were faster when single-letter probes were more likely (low- vs. high-proportion full-word probe). These findings are consistent with the notion that single-letter performance depends on expectations about the likelihood of receiving a single-letter probe, with faster RTs in the low- than in the highproportion full-word probe conditions. Full-word probe probability did not influence full-word probe-typing performance, suggesting that processes mediating full-word typing are automatized (LaBerge \& Samuels, 1974). Finally, results from the lowproportion full-word equal group demonstrated that word advantage effects did not depend on the proportion of primed and unprimed single-letter probes.

\section{Experiment 2: Auditory Primes}

The word advantage effects reported in Experiment 1 are reminiscent of the word superiority effect (Reicher, 1969), in which 
Table 2

Between-Subjects Analysis of Variance Table for Full-Word Reaction Times and Interkeystroke Intervals Across Experiments 1-3

\begin{tabular}{|c|c|c|c|c|c|}
\hline Source & $F$ & $d f \mathrm{~s}$ & $M S E$ & $p$ & $\eta_{\mathrm{p}}^{2}$ \\
\hline \multicolumn{6}{|c|}{ Reaction times } \\
\hline Experiment (E) & 14.04 & 5,90 & $95,877.40$ & $.0001^{*}$ & .44 \\
\hline \multicolumn{6}{|l|}{ Linear contrasts } \\
\hline E1 vs. E2 & 2.08 & 1,90 & $95,877.40$ & .150 & .02 \\
\hline E1 \& E2 vs. E3 & 67.97 & 1,90 & $95,877.40$ & $.0001^{*}$ & .43 \\
\hline Length (L) & 39.27 & 1,90 & 803.64 & $.0001^{*}$ & .30 \\
\hline $\mathrm{E} \times \mathrm{L}$ & 2.06 & 5,90 & 803.64 & .080 & .10 \\
\hline \multicolumn{6}{|c|}{ Interkeystroke intervals } \\
\hline E & 46.73 & 5,90 & $15,290.00$ & $.0001^{*}$ & .72 \\
\hline \multicolumn{6}{|l|}{ Linear contrasts } \\
\hline E1 vs. E2 & 0.00 & 1,90 & $15,290.00$ & .950 & .00 \\
\hline E1 \& E2 vs. E3 & 229.81 & 1,90 & $15,290.00$ & $.0001^{*}$ & .72 \\
\hline $\mathrm{L}$ & 103.40 & 1,90 & 366.16 & $.0001^{*}$ & .53 \\
\hline $\mathrm{E} \times \mathrm{L}$ & 10.31 & 5,90 & 366.16 & $.0001^{*}$ & .36 \\
\hline \multicolumn{6}{|c|}{ Errors } \\
\hline E & 1.59 & 5,90 & 0.00 & .170 & .08 \\
\hline $\mathrm{L}$ & 0.34 & 1,90 & 0.00 & .560 & .00 \\
\hline$E \times L$ & 0.71 & 5,90 & 0.00 & .620 & .04 \\
\hline
\end{tabular}

Note. $\mathrm{E} 1=$ Experiment $1 ; \mathrm{E} 2=$ Experiment 2; E3 $=$ Experiment 3.

letter perception is facilitated for letters appearing within words rather than nonwords. Because visual word primes were used in Experiment 1, it is possible that the word advantage effects were driven by the visual presentation of the letters contained in the prime. In Experiment 2, we addressed the possibility that word advantage effects have a perceptual, rather than motor, locus. To remove the confound, we presented spoken auditory word primes, which lacked the direct presentation of letter information. In this condition, we posited that word advantage effects would not reflect a perceptual benefit for encoding particular letters but would instead reflect a benefit conveyed from processing word information onto the typing procedures employed to execute particular keystrokes.

Experiment 1-3 full word RTs and IKSIs

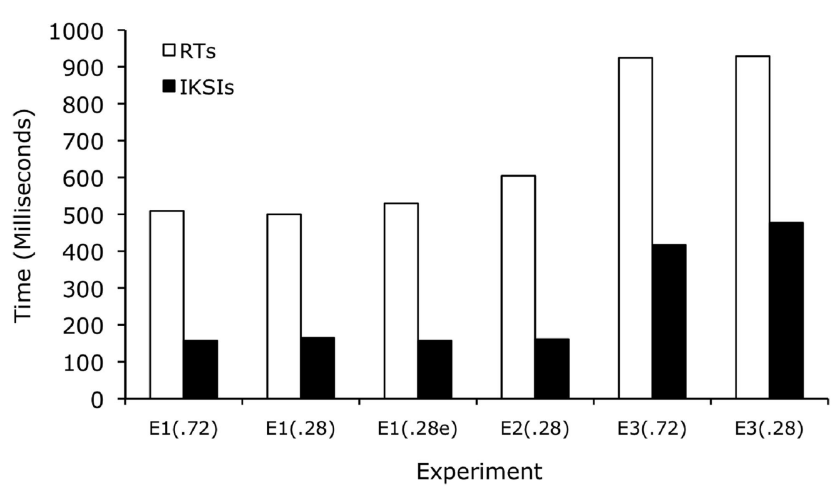

Figure 2. Mean reaction times (RTs) and error rate for full-word and single-letter probes for five- and seven-letter words in Experiment 2. Auditory word primes were used in this experiment.

\section{Method}

Subjects. Subjects were 16 students from Vanderbilt University. All subjects were compensated with course credit or were paid $\$ 12$ per $1 \mathrm{hr}$ of participation. All subjects had normal or corrected-to-normal vision and spoke English as a first language. The mean typing speed for subjects was $67 \mathrm{wpm}$ (range: $32-108$ wpm); mean error rate was .09 (range: 0-.21).

Apparatus and stimuli. We conducted Experiment 2 using the same apparatus as employed in Experiment 1, and the stimuli were selected from the same word list. We synthesized auditory versions of each word using the Apple Macintosh voice synthesizer (Alex voice; Apple Corp., Cupertino, CA). Auditory primes were presented at a comfortable volume level with Sennheiser $\mathrm{eH}$ 150 headphones (Sennheiser Electronics Corp., Old Lyme, CT). Mean length for five-letter words was $544 \mathrm{~ms}$ ( $56 \mathrm{~ms} \mathrm{SD)}$ and 640 ms (66 ms $S D$ ) for seven-letter words.

Procedure. In Experiment 2, we followed the logic of lowproportion full-word probe equal condition in Experiment 1 in which other-letter probes were as frequent as within-word single letter probes. At the beginning of each trial, a fixation cross appeared, followed by the presentation of the auditory prime. The probe was presented visually $150 \mathrm{~ms}$ after the offset of the auditory prime. All other aspects of the procedure were held constant.

\section{Results}

For each subject, RTs in each condition were submitted to an outlier analysis (Van Selst \& Jolicoeur, 1994), which trimmed 2\% of the observations. Mean RTs and error rates collapsed across subjects in each probe proportion condition are depicted graphically in Figure 3. ANOVA tables for the analysis of RTs for single-letter probes are presented in Table 3. Statistical analyses for full-word probes are presented in Table 2. 


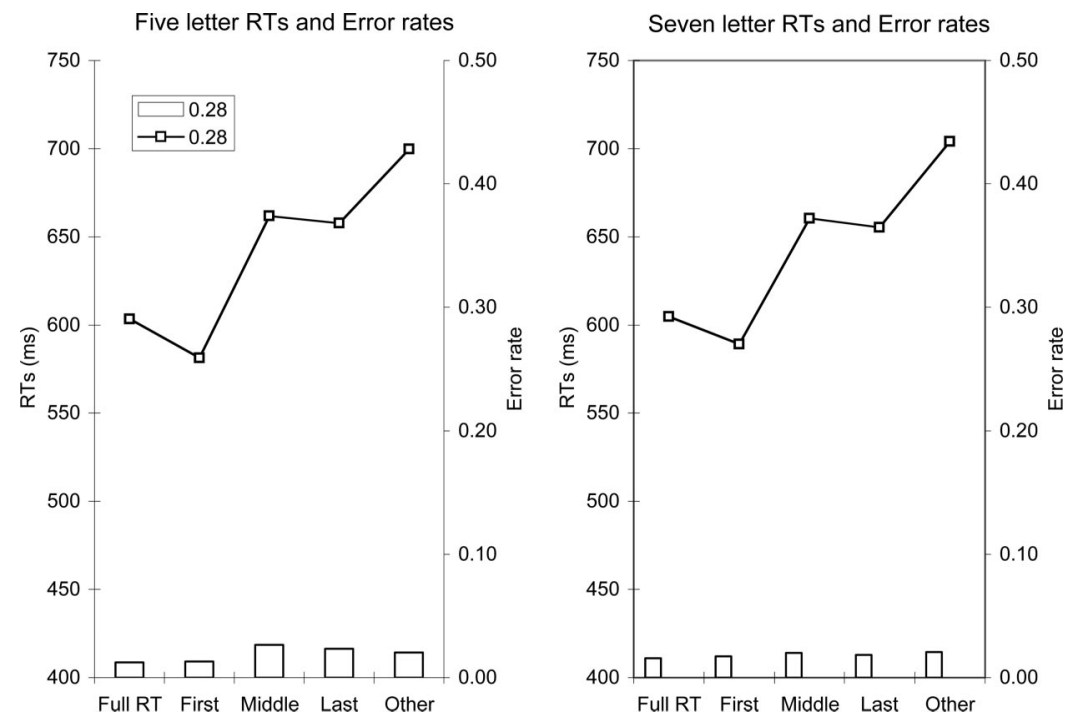

Figure 3. Mean reaction times (RTs) and error rate for full-string and single-letter probes for five- and seven-letter strings for high-proportion full-string probes $(p=.72)$ and low-proportion full-string probes $(p=$ 28) in Experiment 3. Random constant string primes were used in this experiment.

As with Experiment 1, first-letter probes (585 ms) were typed significantly faster than middle-letter probes $(661 \mathrm{~ms})$, which were not different from last-letter probes (657 ms). First-, middle-, and last-letter probes were all typed significantly faster than otherletter probes $(702 \mathrm{~ms})$. These findings replicate the basic pattern of word advantage effects described in Experiment 1. For comparison purposes, we conducted a 2 (Experiment 1: low-proportion fullword equal vs. Experiment 2) $\times 2$ (word length: five vs. seven) $\times$ 4 (probe type: first, middle, last, other) mixed design ANOVA, reported in Table 3. The between-subject experiment factor was not significant and did not interact with the word length or probe type factors.

Table 3

Experiment 2 Analysis of Variance Tables for Single-Letter Probe Reaction Times and Error Rates

\begin{tabular}{|c|c|c|c|c|c|}
\hline Source & $F$ & $d f \mathrm{~s}$ & $M S E$ & $p$ & $\eta_{\mathrm{p}}^{2}$ \\
\hline \multicolumn{6}{|c|}{ Reaction times } \\
\hline Length (L) & 0.34 & 1,15 & 425.93 & .57 & .02 \\
\hline Probe & 69.37 & 3,45 & 1084.54 & $.0001^{*}$ & .82 \\
\hline \multicolumn{6}{|l|}{ Linear contrasts } \\
\hline First vs. middle & 84.9 & 1,45 & 294.57 & $.0001^{*}$ & .65 \\
\hline First vs. last & 75.23 & 1,45 & 294.57 & $.0001^{*}$ & .63 \\
\hline Middle vs. other & 24.61 & 1,45 & 294.57 & $.0001^{*}$ & .35 \\
\hline Last vs. other & 30.27 & 1,45 & 294.57 & $.0001^{*}$ & .40 \\
\hline Middle vs. last & 0.29 & 1,45 & 294.57 & .60 & \\
\hline $\mathrm{L} \times$ Probe & 0.65 & 3,45 & 294.57 & .59 & .04 \\
\hline \multicolumn{6}{|c|}{ Errors } \\
\hline Length & 0.30 & 1,15 & 0.000 & .60 & .02 \\
\hline Probe & 1.04 & 3,45 & 0.000 & .39 & .06 \\
\hline Length $\times$ Probe & 0.66 & 3,45 & 0.000 & .58 & .04 \\
\hline \multicolumn{6}{|c|}{ Between-subjects ANOVA for reaction times in Experiments 1 and 2} \\
\hline Experiment (E) & 0.60 & 1,30 & 117338.79 & .44 & .02 \\
\hline Length $(\mathrm{L})$ & 1.48 & 1,30 & 373.11 & .23 & .05 \\
\hline$E \times L$ & 0.11 & 1,30 & 373.11 & .74 & .00 \\
\hline Probe & 139.94 & 3,90 & 1151.93 & $.0001^{*}$ & .82 \\
\hline $\mathrm{E} \times$ Probe & 0.48 & 3,90 & 1151.93 & .70 & .02 \\
\hline $\mathrm{L} \times$ Probe & 0.45 & 3,90 & 362.80 & .72 & .01 \\
\hline $\mathrm{E} \times \mathrm{L} \times$ Probe & 0.31 & 3,90 & 362.80 & .81 & .01 \\
\hline
\end{tabular}

Note. $\mathrm{ANOVA}=$ analysis of variance. 
Error rates were submitted to a 2 (word length: five vs. seven) $\times$ 4 (probe type: first, middle, last, and other) repeated measures ANOVA with probe type as the sole factor. The main effect of length, probe, and the Length $\times$ Probe interaction were not significant, again indicating that the pattern of RTs was not a result of a speed-accuracy tradeoff.

For full-word probes, mean RT was $603 \mathrm{~ms}$ for five-letter words and $605 \mathrm{~ms}$ for seven-letter words. Mean IKSI was $153 \mathrm{~ms}$ for five-letter words and $169 \mathrm{~ms}$ for seven-letter words. These values were similar to the values for visual probes in Experiment 1.

\section{Discussion}

In Experiment 2, we found significant word advantage effects for first, middle, and last letters using auditory word primes. These findings suggest that the word advantage effects reported in Experiments 1 and 2 were not driven solely by the visual presentation of letters in the word prime. Instead, we argue that the presentation of word information in the prime causes parallel activation of the motor processes responsible for typing particular letters contained within the word.

\section{Experiment 3: Random Letter Strings}

Experiments 1 and 2 suggest that word-level representations cause parallel activation of keystroke execution processes for each letter in the primed word. Experiment 2 was devised to address the concern that word advantage effects were driven by the visual presentations of letters in the word prime. In Experiment 3, we took a different tack in order to remove the confound between word and letter levels of the prime. Rather than presenting words as primes, we visually presented random strings of consonants. This manipulation removed the word status of the primes but maintained the presentation of individual letters.

If the word advantage effect depends on word-level representations, then we would expect little or no facilitation for single-letter probes that were primed by random letter strings. On the other hand, if the pattern of facilitation depends on letter-level representations, then we would expect a similar pattern of facilitation for first, middle, and last letters that were primed by random letter strings.

Experiment 3 was designed in the same fashion as Experiment 1. The only difference was that random strings of consonants were employed in place of word primes. Following Experiment 1, Experiment 3 involved a similar between-subjects full-word string proportion manipulation. The high-proportion full-word-string condition included .72 random-letter-string probes and .28 singleletter probes; and the low-proportion full-word-string condition included .28 random-letter-string probes and .72 single-letter probes.

\section{Method}

Subjects. Subjects were 32 students from Vanderbilt University (16 subjects were assigned to each of the proportion conditions). All subjects were compensated with course credit or were paid $\$ 12$ per $1 \mathrm{hr}$ of participation. All subjects had normal or corrected-to-normal vision and spoke English as a first language. The mean typing speed for subjects in the high-proportion full- string condition was 59 wpm (range: $26-85$ wpm); mean error rate was .09. Mean typing speed for subjects in the low-proportion full-string condition was $68 \mathrm{wpm}$ (range: 44-96 wpm); mean error rate was .08 (range: .04-.14).

Apparatus and stimuli. We conducted Experiment 3 using the same apparatus as employed in Experiment 1. The stimuli in Experiment 3 were changed from words to visually presented random letter strings. All of the random letter strings were made up of consonants and were constructed such that no letter was repeated across a given string and that each letter appeared in each position with approximately equal frequency. Other letter probes were always consonants. The average relative letter frequency for both primed and unprimed letters was 3.8\%. For each subject, 400 five-letter strings and 400 seven-letter strings were created.

Procedure. In Experiment 3, we used the same procedures as Experiment 1. The only difference was that the between-subject manipulation of full-word probe proportion was restricted to two conditions rather than the three employed in Experiment 1. In the high-proportion full-string probe condition, subjects received $72 \%$ full-string probes and $28 \%$ single-letter probes ( $7 \%$ for each of the first-, middle-, last-, and other-probe types). In the low-proportion full-string probe condition, subjects received $28 \%$ full-string probes and $72 \%$ single-letter probes $(18 \%$ for each of the first-, middle-, last-, and other-letter probe types). All other aspects of the design and procedure were held constant.

\section{Results}

Mean RTs and error rates by probe condition, word length, and proportion condition are displayed graphically in Figure 4. For each subject, single-letter probe RTs in each condition were submitted to an outlier analysis (Van Selst \& Jolicoeur, 1994), which trimmed $3 \%$ of the observations. Probe RTs and error rates were submitted to 2 (proportion full string: low vs. high) $\times 2$ (word length: five vs. seven) $\times 4$ (probe type: first, middle, last, other) mixed design repeated measures ANOVAs. Summary tables for the ANOVAs are presented in Table 4.

The most important question was whether primed single-letter probes were typed faster than unprimed single-letter probes. When collapsed across the proportion conditions, RTs for first-letter probes $(655 \mathrm{~ms})$ were shorter than those for middle- $(715 \mathrm{~ms})$, last(725 ms), and other-letter probes (720 ms). RTs for middle- and last-letter probes were not significantly shorter than those for other-letter probes. The probe factor did not interact with the proportion manipulation but did interact with string length. For seven-letter strings, RTs for last-letter probes $(731 \mathrm{~ms})$ were significantly longer than those for both middle- $(707 \mathrm{~ms})$ and otherletter $(716 \mathrm{~ms})$ probes. This pattern was not observed for five-letter strings. No other higher order interactions were significant. To summarize, the random consonant string conveyed an advantage for typing the first letter in the prime string but did not convey an advantage for typing the middle or last letter in the prime string.

The proportion manipulation did not influence the pattern of first-letter advantage effects; however, Figure 4 demonstrates that the proportion manipulation again influenced typing performance for the single-letter probes. To examine this finding, we focused on other-letter probes. RTs to type other-letter probes were slower in high- $(769 \mathrm{~ms})$ than in low- $(671 \mathrm{~ms})$ proportion full-string conditions, $t(120)=2.71$. As with Experiment 1, we interpret this 
Five letter RTs and Error rates

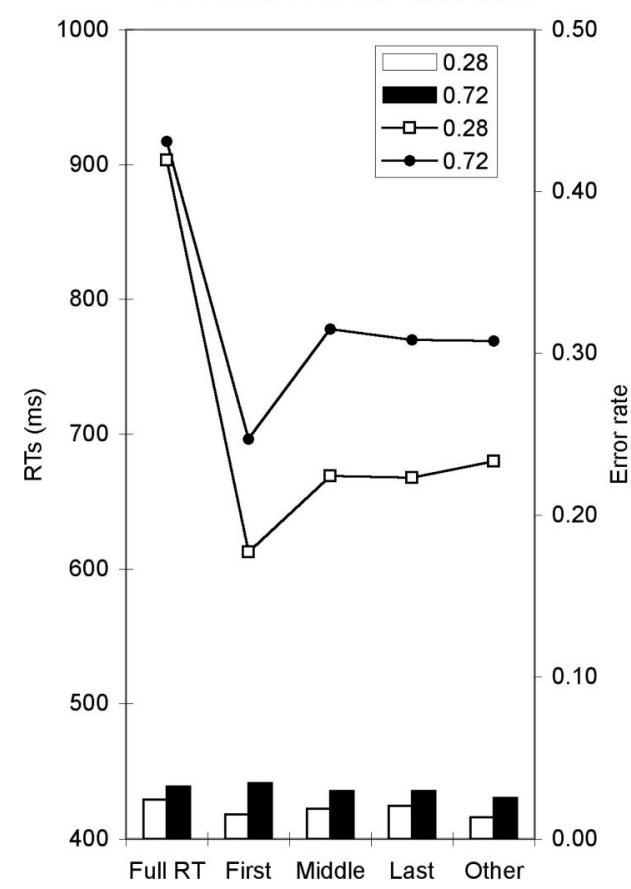

Seven letter RTs and Error rates

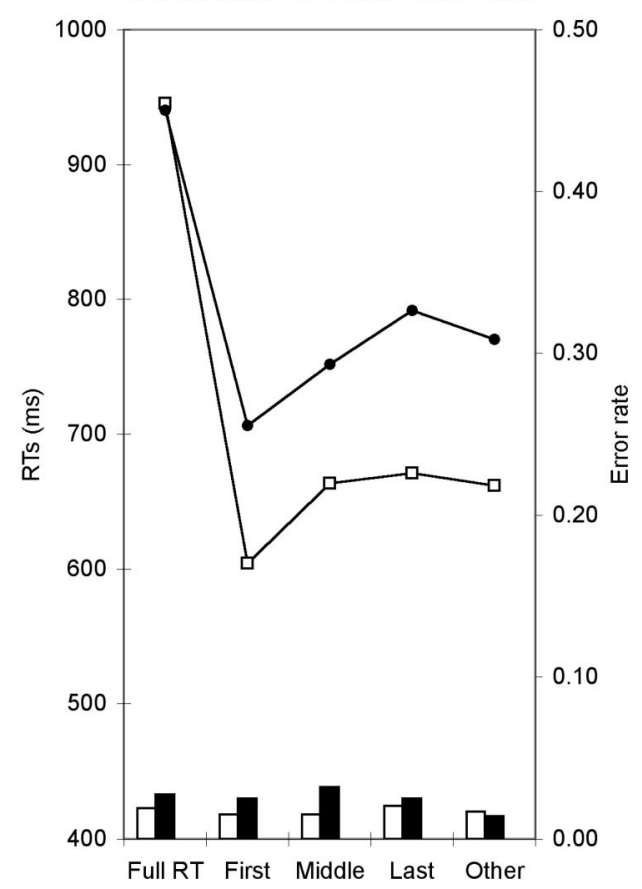

Figure 4. Mean reaction times (RTs) and interkeystroke intervals (IKSIs) for full-word and string probes, collapsed across word length, for all proportions of full-word probe conditions in Experiments (E) 1, 2, and 3: .72 refers to the high-proportion full-word probe conditions, .28 refers to the low-proportion full-word probe conditions, and .28e refers to low-proportion full-word probe condition where the probability of received a primed or other letter probe was equal (e).

finding as evidence that expectations for receiving a single-letter probe can influence RTs to single-letter probes.

Error rates were submitted to a 2 (proportion full string: low vs. high) $\times 2$ (word length: five vs. seven) $\times 4$ (probe type: first, middle, last, other) mixed-design repeated measures ANOVA, reported in Table 4. There were no significant effects, indicating that the observed RT differences were not driven by a speedaccuracy tradeoff.

We submitted RTs and IKSIs for full-string probes to 2 (string length: five vs. seven) $\times 2$ (proportion full string: high vs. low) mixed design repeated measures ANOVAs (see Table 2). Planned comparisons revealed no significant difference between first-keypress RTs in the high- (929 ms) and low- (924 ms) proportion full-string conditions. Similarly, there was no significant difference between IKSIs in the high (449 ms) and low (395 ms) full-string conditions. There was a main effect of string length for IKSIs, $F(1,30)=56.99, M S E=731.92$. IKSIs were faster for five- $(396 \mathrm{~ms})$ than for seven- $(447 \mathrm{~ms})$ letter strings. These findings indicate that subjects' expectations to type a full-string probe had little influence over performance on typing full strings.

We compared RTs and IKSIs for full-string probes with RTs and IKSIs for full-word probes in 2 (length: five vs. seven) $\times 5$ (Experiment: 1: high-, low-, and low-equal proportion full-word probes; Experiment 2: low-equal proportion full-word probes; and Experiment 3: high- and low-proportion full-string probes) ANOVAs. The summary table and contrasts appear in Table 2. The mean RTs and IKSIs appear in Figure 2. RTs and IKSIs were significantly longer in Experiment 3 than in Experiments 1 and 2. These results are consistent with previous work demonstrating that RTs and IKSIs are slowed as the word structure of the to-be-typed string is degraded (Larochelle, 1983; Shaffer \& Hardwick, 1968; Sternberg, Knoll, \& Wright, 1978).

\section{Discussion}

In Experiment 3, we preserved the visual presentation of letters but removed the word status of the prime. As a result, the word advantage effect for the middle and last letters was not observed. Instead, Experiment 3 demonstrated only first-letter advantage effects, which suggests that word advantage effects in Experiment 1 were driven by word-level representations as opposed to letterlevel representations.

All experiments produced larger advantages for first-letter probes than for middle- and last-letter probes, and this first-letter advantage did not depend on the visual or auditory format of the word prime or the word status of the prime. The inner-outer loop theory suggests that the inner loop translates information from the outer loop into keystrokes one chunk at a time. When the outer loop processes words, it passes the entire word as a single chunk to the inner loop; when the outer loop processes random letter strings, it passes each letter as a single chunk to the inner loop. We assume that random letter strings were represented as five or seven chunks in verbal short term memory and passed one chunk (one letter) at a time to the inner loop. Thus, the first letter would be 
Table 4

Experiment 3 Analysis of Variance Tables for Single-Letter Probe Reaction Times and Error Rates

\begin{tabular}{|c|c|c|c|c|c|}
\hline Source & $F$ & $d f \mathrm{~s}$ & $M S E$ & $p$ & $\eta_{\mathrm{p}}^{2}$ \\
\hline \multicolumn{6}{|c|}{ Reaction times } \\
\hline Proportion $(\mathrm{P})$ & 4.94 & 1,30 & $130,807.95$ & $.03^{*}$ & .14 \\
\hline Length (L) & 0.41 & 1,30 & $1,200.94$ & .52 & .01 \\
\hline$P \times L$ & 1.04 & 1,30 & $1,200.94$ & .32 & .03 \\
\hline Probe & 49.60 & 3,90 & $1,401.78$ & $.0001^{*}$ & .62 \\
\hline $\mathrm{P} \times$ Probe & 0.72 & 3,90 & $1,401.78$ & .54 & .02 \\
\hline $\mathrm{L} \times$ Probe & 3.63 & 3,90 & 659.61 & $.02^{*}$ & .11 \\
\hline \multicolumn{6}{|c|}{ Contrasts for five-letter words } \\
\hline First vs. middle & 115.80 & 1,90 & 659.61 & $.0001^{*}$ & .56 \\
\hline First vs. last & 100.81 & 1,90 & 659.61 & $.0001^{*}$ & .53 \\
\hline Middle vs. other & 0.01 & 1,90 & 659.61 & .91 & .00 \\
\hline Last vs. other & 0.69 & 1,90 & 659.61 & .41 & .01 \\
\hline Middle vs. last & 0.52 & 1,90 & 659.61 & .48 & .01 \\
\hline \multicolumn{6}{|c|}{ Contrasts for seven-letter words } \\
\hline First vs. middle & 66.46 & 1,90 & 659.61 & $.0001^{*}$ & .42 \\
\hline First vs. last & 140.68 & 1,90 & 659.61 & $.0001^{*}$ & 61 \\
\hline Middle vs. other & 1.75 & 1,90 & 659.61 & .19 & .02 \\
\hline Last vs. other & 5.69 & 1,90 & 659.61 & $.02^{*}$ & .06 \\
\hline Middle vs. last & 13.75 & 1,90 & 659.61 & $.0001^{*}$ & .13 \\
\hline $\mathrm{P} \times \mathrm{L} \times$ Probe & 2.35 & 3,90 & 659.61 & .08 & .07 \\
\hline \multicolumn{6}{|c|}{ Errors } \\
\hline $\mathrm{P}$ & 3.00 & 1,30 & 0.003 & .09 & .09 \\
\hline $\mathrm{L}$ & 0.96 & 1,30 & 0.001 & .34 & .03 \\
\hline $\mathrm{P} \times \mathrm{L}$ & 1.15 & 1,30 & 0.001 & .29 & .04 \\
\hline Probe & 0.90 & 3,90 & 0.001 & .44 & .03 \\
\hline $\mathrm{P} \times$ Probe & 0.67 & 3,90 & 0.001 & .58 & .02 \\
\hline $\mathrm{L} \times$ Probe & 0.07 & 3,90 & 0.001 & .98 & .00 \\
\hline $\mathrm{P} \times \mathrm{L} \times$ Probe & 0.45 & 3,90 & 0.001 & .72 & .01 \\
\hline
\end{tabular}

passed to the inner loop and that would activate the representation of the corresponding keystroke. No other letters would be passed until the first letter was typed, so there would be no activation in the inner loop (and thus, no priming) for letters beyond the first.

The explanation of first-letter advantage effects in Experiment 1 and 2, which employed word primes, is not as straightforward. We assume that words are passed as single chunks to the inner loop and this activates all of the letters in parallel. One possibility is that a serial ordering process weights the activation of letters to set priorities for keystroke execution (Rumelhart \& Norman, 1982; also see Dell, Burger, \& Svec, 1997). On this view, the serial ordering process weights the first letter more than the others, and this extra weight produces the first letter advantage effects found in Experiments 1 and 2.

Alternatively, the first-letter advantage effect for Experiment 1 and 2 could be explained by hierarchical representations within the outer loop. The outer loop may represent both word and letter information. On this view, the outer loop passes word and letter chunks to the inner loop, and the first letter receives activation from both chunks, whereas the middle and last letter only receive activation from the word chunk. The combined influence of word and letter chunks could explain the first-letter advantage effect in Experiments 1 and 2. Although from the present results, we cannot distinguish between these accounts of the first-letter advantage effect, the interpretation of the word advantage effect for middle and last letters is clear. Word primes activate middle and last letters in parallel.

\section{General Discussion}

Theories of typing assume that hierarchical processing loops control typing at the level of words, letters, and motor responses (John, 1996; Logan \& Crump, 2009; Rumelhart \& Norman, 1982; Salthouse, 1986; Shaffer, 1976; Wu \& Liu, 2008). Theories commonly assume that word-level information initiates parallel activation of constituent letters and their motor responses for keystroke execution. In previous research, letter information has been shown to automatically activate keystroke execution for particular letters (Rieger, 2004, 2007); however, the parallel activation assumption has not been tested directly (but as discussed in the introduction, see Flanders \& Soechting, 1992; Gentner, 1983; Gentner et al., 1980; Logan, 2003). In the current experiments, we provided a direct test of the parallel activation assumption by developing a novel single-letter probe technique. Subjects were presented with a word or nonword prime and then probed to type the full word, a single letter contained in the probe string, or another letter not contained in the prime string.

Experiments 1-3 provided evidence that words activate their constituent letters in parallel. Experiment 1 demonstrated that a visually presented word prime facilitates typing responses for the first, middle, and last letters in the prime, relative to other letters not in the prime. Experiments 2 and 3 ruled out the possibility that the word advantage effects reflected benefits to the perceptual processing of letters displayed in the prime. In Experiment 2, we 
employed auditory word primes, which eliminated the visual presentation of letters, and found word advantage effects for the first, middle, and last letters. In Experiment 3, we employed visual random consonant string primes, which presented letters but not words, and found a first letter advantage effect but no within-string advantage effect for the middle and last letters. Taken together, these results demonstrate that word-level representations cause parallel activation of letters and keystrokes contained in the word. All experiments demonstrated first letter advantage effects, whereby RTs were consistently shorter for first-letter probes than middle- and last-letter probes. The first letter advantage effect suggests that letter-level information also facilitates keystroke execution (also see Rieger, 2004; 2007). Last, we provided converging evidence of the importance of word units in controlling typing by finding faster RTs and IKSIs for visual and auditory words than random consonant strings (Larochelle, 1983; Shaffer \& Hardwick, 1968; Sternberg, Knoll, \& Wright, 1978).

We conducted the current experiments to test the parallel activation assumption that words cause the parallel activation of constituent keystrokes. The first-, middle-, and last-letter advantage effects in Experiments 1 and 2 provide direct support for the parallel activation hypothesis. An alternative serial-activation account would predict a gradient of priming across the letters in the word. If activation spreads serially across the letters in the word, we would expect a linear trend with the most activation for the first letter, less activation for the middle letter, and even less activation for the last letter. In addition, we would expect that the middle- and last-letter advantage effects would interact with word length. Because seven-letter words are longer, middle and last letters in these words should take longer to prime, and therefore, we would expect smaller middle- and last-letter advantage effects for seven-letter words than for five-letter words. The present data are not consistent with a serial activation account. Specifically, we found no differences between middle- and last-letter advantage effects and no interaction with word length. Instead, the present data are strongly consistent with the parallel activation hypothesis.

The evidence that words activate letters in parallel is consistent with other studies that provide less direct evidence for parallel processing (Flanders \& Soechting, 1992; Gentner, 1983; Gentner et al., 1980; Logan, 2003). It confirms specific predictions from Rumelhart and Norman's (1982) computational model of typing and is broadly consistent with theories of typing that propose hierarchical processing (John, 1996; Salthouse, 1986; Shaffer, 1976; Wu \& Liu, 2008). The evidence of parallel activation is also consistent with our inner-outer loop theory of typing (Logan \& Crump, 2009) and suggests that the two loops deal with different units of information. Logan and Crump (2009) showed that the outer loop does not know which hands execute which keystrokes. The present results explain why: The outer loop is concerned with words and not with the keystrokes with which they are composed.

The consistent evidence for hierarchical processing in the control of typewriting contrasts with the ambiguous evidence for hierarchical processing in other examples of cognitive control (Botvinick \& Plaut, 2004; Shallice \& Cooper, 2000; Schneider \& Logan, 2006, 2007). For example, Shallice and Cooper (2000) accounted for errors in a coffee-making task with a model in which hierarchically controlled schemas are assumed, whereas Botvinick and Plaut (2004) accounted for the same data with a model in which a nonhierarchical recurrent network is assumed. We suggest three reasons why the evidence for hierarchical processing may be clearer in typing.

First, typing is dependent on pre-existing language and reading skills. A survey of our subjects indicated that most of them began typing in elementary or middle school, well after their language skills had developed and after they were proficient readers. They already had the ability to think and converse in words. To acquire skill at typing, they had to learn to translate words into series of keystrokes. That is, the higher levels of the hierarchy were well established; they had to learn to translate them into novel lower level processes. By contrast, coffee making is a relatively novel task that recruits pre-existing motor skills, like reaching, grasping, tearing, and pouring. The higher levels of the hierarchy may be built upon well-established lower level skills (Shallice \& Cooper, 2000), or the higher levels may be concatenations of pre-existing low-level skills (Botvinick \& Plaut, 2004).

Second, speed is an important characteristic of typing skill, and hierarchical processing may be necessary to achieve the required speed. Speaking (120-200 wpm) and reading (250-350 wpm; Rayner \& Clifton, 2009) are both substantially faster than skilled typing (50-100 wpm). A fundamental problem in typing is to type as fast as one can read or think (speak to oneself); the fingers must catch up to the tongue or eye. Typists appear to solve this problem by cascading the component processes so there is substantial parallel processing. Indeed, high-speed video of skilled typing shows that movements for successive keystrokes overlap substantially in time (Gentner et al., 1980). Speed is not as important an issue in tasks like coffee making, so there may be less incentive (and pressure) to process hierarchically.

Third, typing requires the coordination of separate perceptual, cognitive, and motor systems and hierarchical processing may be a natural solution to the problem of coordinating separate systems. The cognitive system works with words while the motor system works with keystrokes, and each word requires several keystrokes ("I" and "a" are exceptions). It may be advantageous to let the motor system control the sequencing and execution of keystrokes (Lashley, 1951). By contrast, in coffee making, the mapping of cognitive units onto motor "units" may be more one to one (e.g., "Get the sugar" involves a single reach for the sugar bowl). In more cognitive tasks, higher and lower level processes may be part of the same system (e.g., memory retrieval; Schneider \& Logan, 2006, 2007), so it may be less advantageous to organize the processes hierarchically.

This analysis focuses primarily on the processes required for skilled typing. The representations in typing are clearly hierarchical (sentences, words, letters, keystrokes) but so are the representations in coffee making. One point of the analysis is that hierarchical representations do not necessitate hierarchical processes. Cooper and Shallice (2000) and Botvinick and Plaut (2004) agreed that coffee making may involve hierarchical representations. They disagreed on the question of whether hierarchical representations require hierarchical processes. Understanding the conditions under which they do is an important direction for future research. We suggest that typing may be a useful model system because both representations and processes are hierarchical. 


\section{References}

Botvinick, M. M., \& Plaut, D. C. (2004). Doing without schema hierarchies: A recurrent connectionist approach to normal and impaired routine sequential action. Psychological Review, 111, 395-429. doi: 10.1037/0033-295X.111.2.395

Dell, G. S., Burger, L. K., \& Svec, W. R. (1997). Language production and serial order: A functional analysis and a model. Psychological Review, 104, 123-147. doi:10.1037/0033-295X.104.1.123

Flanders, M., \& Soechting, J. F. (1992). Kinematics of typing: Parallel control of the two hands. Journal of Neurophysiology, 67, 1264-1274.

Gentner, D. R. (1983). Keystroke timing in transcription typing. In W. E. Cooper (Ed.), Cognitive aspects of skilled typewriting (pp. 95-120). New York, NY: Springer-Verlag.

Gentner, D. R., Grudin, J., \& Conway, E. (1980). Finger movements in transcription typing (Tech. Rep. 8001). La Jolla, CA.: University of California at San Diego, Center for Human Information Processing.

John, B. E. (1996). TYPIST: A theory of performance in skilled typing. Human Computer Interaction, 11, 321-355. doi:10.1207/ s15327051hci1104_2

Kucera, H., \& Francis, W. N. (1967). Computational analysis of presentday American English. Providence, RI: Brown University Press.

Laberge, D., \& Samuels, S. J. (1974). Toward a theory of automatic information processing in reading. Cognitive Psychology, 6, 293-323.

Larochelle, S. (1983). A comparison of skilled and novice performance in discontinuous typing. In W. E. Cooper (Ed.), Cognitive aspects of skilled typewriting (pp. 67-94). New York, NY: Springer-Verlag.

Lashley, K. S. (1951). The problem of serial order in behavior. In L. A. Jeffress (Ed.), Cerebral mechanisms in behavior (pp. 112-131). New York, NY: Wiley.

Logan, G. D. (2003). Simon-type effects: Chronometric evidence for keypress schemata in typewriting. Journal of Experimental Psychology: Human Perception and Performance, 29, 741-757. doi:10.1037/00961523.29.4.741

Logan, G. D., \& Crump, M. J. C. (2009). The left hand doesn't know what the right hand is doing: The disruptive effects of attention to the hands in skilled typewriting. Psychological Science, 10, 1296-1300.

Logan, G. D., \& Zbrodoff, N. J. (1998). Stroop-type interference: Congruity effects in color naming with typewritten responses. Journal of Experimental Psychology: Human Perception and Performance, 24, $978-$ 992. doi:10.1037/0096-1523.24.3.978

Lu, C.-H., \& Proctor, R. W. (1995). The influence of irrelevant location information on performance: A review of the Simon and spatial Stroop effects. Psychonomic Bulletin \& Review, 2, 174-207.

McNamara, T. P. (2005). Semantic priming: Perspectives from memory and word recognition. New York, NY: Taylor \& Francis.

Miller, G. A., Galanter, E., \& Pribram, K. H. (1986). Plans and the structure of behavior. New York, NY: Adams-Bannister-Cox.

Neely, J. H. (1977). Semantic priming and retrieval from lexical memory: Roles of inhibitionless spreading activation and limited-capacity attention. Journal of Experimental Psychology: General, 106, 226-254.

Nelson, D. L., McEvoy, C. L., \& Schreiber, T. A. (1998). The University of South Florida word association, rhyme, and word fragment norms. Retrieved from http://www.usf.edu/FreeAssociation/

Ostry, D. J. (1980). Execution-time movement control. In G. E. Stelmach \& J. Requin (Eds.), Tutorials in motor behavior (pp. 457-468). Amsterdam, the Netherlands: North-Holland. doi:10.1016/S01664115(08)61962-7

Rayner, K., \& Clifton, C. (2009). Language processing in reading and speech perception is fast and incremental: Implications for event-related potential research. Biological Psychology, 80, 4-9. doi:10.1016/ j.biopsycho.2008.05.002

Reicher, G. M. (1969). Perceptual recognition as a function of meaningfulness of stimulus material. Journal of Experimental Psychology: General, 81, 275-280.

Rieger, M. (2004). Automatic keypress activation in skilled typing. Journal of Experimental Psychology: Human Perception and Performance, 30, 555-565. doi:10.1037/0096-1523.30.3.555

Rieger, M. (2007). Letters as visual action-effects in skilled typing. Acta Psychologica, 126, 138-153. doi:10.1016/j.actpsy.2006.11.006

Rumelhart, D. E., \& Norman, D. A. (1982). Simulating a skilled typist: A study of skilled cognitive-motor performance. Cognitive Science, 6 , 1-36. doi:10.1207/s15516709 $\operatorname{cog} 0601 \_1$

Salthouse, T. A. (1986). Perceptual, cognitive, and motoric aspects of transcription typing. Psychological Bulletin, 99, 303-319. doi:10.1037/ 0033-2909.99.3.303

Schneider, D. W., \& Logan, G. D. (2006). Hierarchical control of cognitive processes: Switching tasks in sequences. Journal of Experimental Psychology: General, 135, 623-640. doi:10.1037/0096-3445.135.4.623

Schneider, D. W., \& Logan, G. D. (2007). Retrieving information from a hierarchical plan. Journal of Experimental Psychology: Learning, Memory, and Cognition, 33, 1076-1091. doi:10.1037/0278-7393.33.6.1076

Shaffer, L. H. (1975). Control processes in typing. Quarterly Journal of Experimental Psychology, 27, 419-432. doi:10.1080/14640747508400502

Shaffer, L. H. (1976). Intention and performance. Psychological Review, 83, 375-393. doi:10.1037/0033-295X.83.5.375

Shaffer, L. H., \& Hardwick, J. (1968). Typing performance as a function of text. Quarterly Journal of Experimental Psychology, 20, 360-369. doi:10.1080/14640746808400175

Shallice, T., \& Cooper, R. (2000). Contention scheduling and the control of routine activities. Cognitive Neuropsychology, 17, 297-338. doi: $10.1080 / 026432900380427$

Sternberg, S., Knoll, R. L., \& Wright, C. E. (1978). Experiments on temporal aspects of keyboard entry. In J. P. Duncanson (Ed.), Getting it together: Research and applications in human factors (pp. 28-50). Santa Monica, CA: Human Factors Society.

Sternberg, S., Monsell, S., Knoll, R. L., \& Wright, C. E. (1978). The latency and duration of speech and typewriting. In G. E. Stelmach (Ed.), Information processing in motor control and learning (pp. 117152). New York, NY: Academic Press.

Van Selst, M., \& Jolicoeur, P. (1994). A solution to the effect of sample size on outlier elimination. Quarterly Journal of Experimental Psychology: Human Experimental Psychology, 47(A), 631-650.

Weingarten, R., Nottbusch, G., \& Will, U. (2004). Morphemes, syllables, and graphemes in written word production. In T. Pechmann \& C. Habel (Eds.), Language production (pp. 529-572). Berlin, Germany: Mouton de Gruyter.

Will, U., Nottbusch, G., \& Weingarten, R. (2006). Linguistic units in word typing: Effects of word presentation modes and typing delay. Written Language \& Literacy, 9, 153-176. doi:10.1075/wll.9.1.10wil

Wu, C., \& Liu, Y. (2008). Queuing network modeling of transcription typing. ACM Transactions on Computer-Human Interaction, 15, Art. 6. doi: $10.1145 / 1352782.1352788$

Received June 24, 2009

Revision received October 6, 2009

Accepted October 21, 2009 